\title{
The Potential Role of Child Life Specialists in the South African Healthcare Sector
}

\author{
Jacqueline Kim Bezuidenhout. MBCHB (UP); DCH (SA); FCPAED (SA); Certificate of Development(SA); \\ MSc Neurodevelopment(Wits). http://orcid.org/0000-0002-530 I-977 I \\ Senior lecturer, Department of Paediatrics, University of the Witwatersrand, Johannesburg, South Africa.
}

\author{
Joanne Potterton. BSc Physiotherapy (Wits), MSc Physiotherapy (Wits), PhD (Wits). \\ http://orcid.org/0000-0002-8020-274X \\ Personal Professor, Department of Physiotherapy, University of the Witwatersrand.
}

\author{
*Janine van der Linde. B. OT (UFS), MSc. OT (Wits) PhD (Wits). http://orcid.org/0000-0003-1067-9494 \\ Lecturer, Department of Occupational Therapy, University of Witwatersrand, Johannesburg, South Africa.
}

Introduction: Child Life Specialists (CLS) are an integral component of paediatric health care services in many developed countries. They assist in bridging the communication between the healthcare team and the child's parents and family. Their primary aim is to minimise the potential trauma the child and their caregivers may experience from being hospitalised. This field of psychosocial support for ill and hospitalised children has been growing over the past six decades with CLS services currently being offered at most North American paediatric hospitals.

Methods: By using developmentally appropriate techniques, and equipped with a foundation of medical knowledge, the CLS primarily uses play to relieve anxiety and to educate the child. Play is a meaningful activity that allows the healthy development of the child's emotional, behavioural and social development. The three forms of play that may be utilised by the CLS include therapeutic play, normative play and medical play. Non-pharmacological pain management is another vital area within which the CLS is involved.

Conclusion: The South African Department of Health recognises the importance of addressing the psychosocial welfare of the ill child and they have provided guidelines highlighting the psychosocial needs of children affected and infected with HIV. South Africa has many children accessing health services daily and is suitably positioned to initiate CLS in both public and private healthcare facilities. Improving the healthcare experience for the paediatric healthcare user population is crucial in ensuring their emotional and psychological well-being.

\section{INTRODUCTION}

The Child Life Specialist (CLS) is an integral component of paediatric healthcare services in many developed countries including the United States of America, the United Kingdom and New Zealand'. Their role is multifold, but their primary aim is to promote the well-being of infants, children and adolescents and to minimise the trauma that may result from illness, hospitalisation and the accompanying medical procedures that the child may endure. The CLS also serves to support the family and siblings, whilst providing them with information, so that they may better understand and cope with their loved one's condition and make well-informed decisions about future care.

Prior to the 1950's, no child life services were formally available in North America. The closest form of intervention included play programmes that were offered at certain hospitals. The first Child Life and Education division was established in 1955 at the Cleveland City Hospital by Emma Plank, under the direction of Dr Frederick C. Robbins. Emma Plank was an educator and is affectionately regarded as 'the mother of Child Life'. Her role was to address the psychological, social and educational needs of the children who were hospitalised with chronic conditions, and her book "Working with children in hospitals" was instrumental in educating her peers about the unique needs of these children².

The child life movement grew over the next couple of decades as the positive impact on the well-being of hospitalised children became increasingly evident. In 1982, a non-governmental organisation called the Child Life Council (CLC) was established. The CLC was rebranded in 2016 to the Association of Child Life Professionals (ACLP), in order to advance the field of Child Life as well as maintain the standard of service providers globally. At present, the ACLP has more than 6000 registered members who include CLS, as well as educators and health care providers'.

To be eligible for registration as a certified CLS the individual must have an undergraduate bachelor's degree in child life, child development or any other closely related field from an accredited academic institution recognised by the USA Department of Education or an international equivalent. The candidate is then required to complete coursework offered by an ACLP-endorsed child life academic programme or complete a postgraduate course offering the required modules. The third component requires the candidate to complete a 480-600-hour internship under the guidance of a certified CLSI. Until now, no academic institute in South Africa has offered this unique and essential course. In 202I, the University of Witwatersrand commenced the MSc in Child Life and Psychosocial Services which offers the post graduate course work to healthcare workers registered with the Health Professional Council of South Africa (HPCSA). It does not currently provide the internship required for full registration with the ACLP. 


\section{COMMENTS}

\section{Role of the Child Life Specialist}

Although the hospital is an environment that is well suited to address the medical, surgical and acute rehabilitation needs of an unwell child, it typically falls short in addressing the associated trauma and anxiety experienced by a hospitalised child. Being in an unfamiliar environment, possibly being separated from your caregiver, as is typical in many South African state hospitals, and experiencing uncomfortable and often painful procedures, may result in considerable trauma to the young child ${ }^{2}$. The CLS works as part of an interdisciplinary, family centered team involving the patient, family, doctors, nurses, social workers, and other health care providers to develop a comprehensive plan of care. CLS contributions to this plan are based on the child's and family's psychosocial needs, their cultural heritage, and responses to the health care experience'. They typically work in hospitals that have paediatric departments, in both the wards and outpatient clinics, as well as in hospices and special schools. Their primary role is to utilise their understanding of a child's development to appropriately communicate with the child and minimise the potentially negative consequences of hospitalisation and ill-health on the child's well-being. The CLS assists in preparing children emotionally and psychologically for potentially painful or stressful procedures, clinic visits and hospitalisations. This is achieved by using several techniques, including education, age-appropriate communication, non-pharmacological inteventions for pain management ${ }^{3}$ and therapeutic and medical play ${ }^{4}$.

CLSs are integral in alleviating the anxiety and distress experienced by the caregivers and family of the child, and are the 'connection' between a busy medical or surgical team and the family. Cuzzocrea et al. ${ }^{5}$ found that the anxiety levels of mothers who had received preoperative psychological preparation prior to their children having surgery, were reduced compared to those mothers who had received none. Informed and educated parents are better equipped to be more emotionally available for their children ${ }^{5}$.

\section{The CLS and Pain Management}

Non-pharmacological pain management strategies have been identified as a key role of CLS'. Interventions for pain and distress management led by the CLSs have proven successful in terms of patient and family experience, staff experience, and cost effectiveness'. Pain management remains a challenge in paediatric care despite recent advances in the field $^{6}$, and this remains an area in which the CLS addresses, using a variety of approaches'. There is a growing body of evidence highlighting the need for non-pharmacological management of pain in infants and young children'. Successful interventions include non-nutritive sucking, swaddling/tucking and holding and rocking. Distraction, for example allowing the child to play on an iPad during a painful procedure, has been reported to be effective in reducing observed and reported pain in a number of clinical settings including emergency departments' ${ }^{\prime}$. Bandstra et $a^{7}$ found that providing information, comfort, reassurance and positive reinforcement were the most widely used non-pharmacological techniques employed to ease pain.

\section{Power of play and the CLS}

Although many healthcare workers incorporate play into their treatments in order to reach therapeutic goals, the CLS places a slightly different emphasis on play. Guiding children through multiple dimensions of play, according to their developmental needs and environmental context, is a core competency of child life practitioners ${ }^{2}$.

Burns-Nader et al. ${ }^{8}$ discuss the different types of play used by CLS including therapeutic play, normative play or medical play, which may be used to provide the child with coping strategies to reduce anxiety, stress and pain or to promote knowledge about medical procedures or to just allow the child to experience some normality in a chaotic situation ${ }^{8}$.

Therapeutic play activities such as puppets, storybooks,arts and crafts or toys where the child and CLS engage in imaginative play, allow the CLS to model coping behaviour through the characters, and give the child the opportunity to express their emotions ${ }^{8}$. Lookabaugh et al. ${ }^{9}$ report that not only does the hospitalised child benefit from therapeutic play, but it also benefits siblings who may also experience anxiety and stress during this time?

An important aspect of therapeutic play is medical play. Medical play is used to promote knowledge regarding the medical equipment and procedure that the child will encounter during their stay in the hospital ${ }^{8}$. The exploration of medical equipment in a structured nonthreatening manner using songs, games and imaginative play, allows the child to reduce their anxiety and the opportunity to gain control over their emotions8. Medical play not only benefits the child but helps reduce stress and improve coping for siblings and parents'.

\section{The CLS and Auxillary programmes}

In addition to using play as a means of decreasing anxiety in a hospitalised child and as a therapeutic modality, the CLS utilises several auxillary programmes to augment the child life services. Some of these programs include therapeutic clowning and animal-assisted therapy. Therapeutic clowns are specially trained clowns who incorporate music, drama, humour and play whilst building relationships and relieving anxiety for the child requiring medical intervention. Animal-assisted therapy is a non-pharmacological intervention that has been used to assist in alleviating anxiety in hospitalised children. The therapeutic animal is typically brought into the ward accompanied by its handler and the child and their family can interact spontaneously with the animal, thus providing a sense of comfort and a reduction in stress. Some of the other interactions that therapeutic animals may be involved within the hospital or outpatient setting, include providing comfort and distraction during nursing, physiotherapy or blood taking procedures. Although the benefits of pet therapy for children who are hospitalised is an area of ongoing research, there appears to be a positive response from the medical personnel and families who have been exposed to the intervention'. The CLS may also work in collaboration with certified play therapists, art therapists and music therapists in the hospital setting who are an essential component of the expressive therapies.

The CLS has many functions to fulfill including providing the child with psychological preparation for possible medical procedures and interventions; assisting with the nonpharmacological management of pain; providing developmentally appropriate stimulation and enjoyment through the various forms of play, and to be a support and source of information for the family. The Committee on Hospital Care and Child Life Council advocate strongly for the inclusion of CLS in hospitals. Including the services of a CLS has shown to reduce the overall length of stay in hospital and decrease the need for sedation and analgesia, thus improving the overall patient and family experience in hospital ${ }^{10}$.

\section{Where does South Africa fit into the equation?}

South Africa is home to $57,779,622$ people at the last population estimate, and is classified as an upper-middle-income economy by the World Bank" . According to the latest figures from Statistics SA 2018 , there were 7,52 million people infected with HIV in $2018^{12}$. The paediatric population of South Africa is burdened with an under five mortality rate of 33.4 per 1000 live births ${ }^{12}$. Although the mortality 
rate has significantly improved over the past decade, it is still above the expected rate of 25 per 1000 live births as recommended by the Sustainable Developmental Goals (SDG) $2030^{13}$. According to United Nations estimates, the leading causes of under five mortality include neonatal conditions, pneumonia, injury, gastroenteritis, AIDS, and congenital abnormalities. With high rates of infection and malnutrition, many children require the services of healthcare professionals in both inpatient and outpatient settings ${ }^{14}$.

South Africa has a healthcare system that is divided into a public sector government system, serving most of the population, and a private sector that is privately financed and utilised by individuals with medical aids. Despite the discrepancy between the two systems with regards to infrastructure and resources, the South African Department of Health recognises the benefit of treating the child holistically. A guideline compiled by the Department of Health was issued to educate the healthcare providers on the psychosocial support needed by children and adolescents infected and affected by HIV/AIDS ${ }^{15}$. These guidelines highlight the importance of addressing the emotional, mental, spiritual and cognitive needs of the child, as these areas impact significantly on a child's physical health. Addressing the psychosocial well-being of a child, substantially impacts on them reaching their full potential ${ }^{15}$.

A number of South African paediatric units have already established the practice of incorporating psychosocial care and well-being into the management of their paediatric patients. Van Niekerk et al. ${ }^{16}$ described some of the enablers of psychosocial recovery in the paediatric burn unit at The Red Cross Children's' War Memorial Hospital in Cape Town, South Africa. Some of these enablers included allowing the family in the ward with the child to help reassure the child, as well as having the nursing staff provide emotional support to the child. They also promoted involving the child in certain aspects of the decision making of their care, thus allowing them to feel some sense of control during a painful and frightening experience ${ }^{16}$. Volunteers who were burn victim survivors also decreased the anxiety levels of children by reading to them and being a role model of survival for the child $^{16}$. This approach follows many of the guiding principles of CLS.

Hendricks and colleagues, described the value of the multidisciplinary psychosocial care team that is functioning in the paediatric oncology unit at a state hospital in the Western Cape ${ }^{17}$. The team is a collaboration between state and private sector practitioners including paediatric oncologists, psychologists, social workers, psychologists, art therapists, religious leaders and palliative care doctors, who are all involved in providing support both medically and emotionally to the child and family ${ }^{17}$.

The aim to improve the health care experience for our paediatric population is critical to ensure their emotional and psychological well-being. Adopting a CLS approach would allow existing efforts to be streamlined and mainstreamed into the holistic care of children in all South African healthcare facilities. The training in child life and paediatric psychosocial care currently offered at Wits will introduce healthcare workers to child life principles which they can incorporate into their work with children. In time, getting CLS recognised by the HPCSA would allow for optimal implementation of child life services in South African healthcare facilities.

\section{RECOMMENDATIONS AND CONCLUSION}

There is a growing body of research evidence, supported by clinical experience, to promote the role of child life specialists in paediatric facilities. South Africa has a large number of children accessing health services daily and is suitably positioned to initiate child life services in both public and private facilities. There is a need for a co-ordinated inter-professional approach to both the training and policy changes that will be needed to introduce child life specialists into our healthcare system.

\section{REFERENCES}

I. Romito B, Jewell J, Jackson M. AAP Committee on hospital care; Association of Child Life Professionals. Pediatrics. 202I; 147(I); https:// doi.org/10.1542/peds.2020-04026 I

2. Thompson RH.The Handbook of Child Life. A Guide for Pediatric Psychosocial Care. Springfield, Illinois: Charles C. Thomas: 2009 (2nd ed, 2018).

3. Association of Child Life Professionals Mission Statement. https://www.childlife.org/the-child-life-profession/mission-valuesvision (accessed 27 October 202I).

4. Lerwick J. An Exploration of the Roles of Certified Child Life Specialists \& Registered Play Therapists in the United States. Int J Pediatr Res. 2019; 5:049. https://doi.org/10.23937/2469-5769/1510049

5. Cuzzocrea F, Gugliandolo MC, Larcan R, et al. A psychological preoperative program: effects on anxiety and cooperative behaviors. Pediatric Anesthesia. 2013; 23(2):

https://doi.org/10.1 I I I/pan. 12100

6. Pillai RR, Racine N, Gennis $\mathrm{H}$, et al. Non-pharmacological management of infant and young child procedural pain. Cochrane database of systematic reviews. 2015 (I2). https://doi.org/10.1002/ebch. I883

7. Bandstra NF, Skinner L, Leblanc $C$, et al. The role of child life in pediatric pain management: a survey of child life specialists. J Pain. 2008;9(4):320-329. https://doi.org/10.1016/j.jpain.2007.11.004

8. Burns-Nader S, Hernandez-Reif M. Facilitating play for hospitalized children through child life services. Children's Health Care. 2016; 45(I), I-2I. https://doi.org/I0.1080/027396I5.2014.948I6I

9. Lookabaugh S, Ballard SM. The Scope and Future Direction of Child Life. Journal of Child and Family Studies. 20I8; 27(6), I72I-I73I. https://doi.org/10.1007/s10826-018-1031-6

13. World Health Organization: Sustainable Development Goals: Health topics. Sustainable Development Goals (who.int) (accessed 27 October 202I).

14. Bamford LJ, McKerrow NH, Barron P, et al. Child mortality in South Africa: Fewer deaths, but better data are needed. South African Medical Journal. 2018; I: s25-s32. https://doi.org/10.7196/samj.2017.v108i3b. 12779.

15. South African Department of Health. Psychosocial support (PSS) for children and adolescents infected and affected by HIV and AIDS. Psychological-Support-PSS-for-Children-and-Adolescents-Infectedand-Affected-by-HIV-and-AIDS.pdf (teampata.org) (accessed 27 October 202I)

16. Van Niekerk A, Jacobs R, Hornsby N, et al. Enablers of psychosocial recovery in pediatric burns: perspectives from the children, parents and burn recovery support staff. BMC Pediatrics. 2020;20(I):289. Published 2020 Jun 9. https://doi.org/10.1 I86/s/2887-020-02180-z

17. Hendricks M, Steenveld CM, Thompson V, et al. Building a psychosocial and spiritual care service for children with cancer and their families. South African Journal of Oncology. 2019;3(0), a52. https://doi.org/10.4102/sajo.v3i0.52

Corresponding Author

*Janine van der Linde

Email: Janine.VanDerLinde@wits.ac.za 